WS9-A06

\title{
Anisotropic Inversion of Towed Streamer EM Data in Shallow Waters
}

\author{
J. Mattsson* (Petroleum Geo-Services)
}

\section{SUMMARY}

Towed streamer electromagnetic (EM) data along a survey line at the Alvheim Boa oil field in the North Sea has been inverted using an open-source 2.5D inversion code. The electric field data was acquired in 4 $\mathrm{kn}$ with a single vessel using a horizontal bipole source at $10 \mathrm{~m}$ depth and densely populated electrode pair receivers housed in a towed streamer towed at $50 \mathrm{~m}$ depth in water depths between 110 and $125 \mathrm{~m}$. The inversion algorithm is based on a parallel adaptive $2.5 \mathrm{D}$ finite element algorithm and uses a regularized variant of a Gauss-Newton minimization algorithm. This inversion method has proven to be fast and efficient for and suitable for towed streamer EM data. In this case, the resulting anisotropic resistivity cross section corresponds well with the high resistive part of the sand layer in the vicinity of the reservoir depocenter. The low noise in the frequency response data and the low navigation and measurement uncertainties made it possible to obtain a final misfit as low as $2 \%$. The towed streamer EM technology has proven to be a robust and useful CSEM method in shallow waters. The densely sampled frequency response data is suitable for anisotropic inversion. 


\section{Amsterdam' '14}

\section{Introduction}

The first available towed streamer EM system was tested in its final form in October 2012. The similarities to towed streamer seismic acquisition are obvious, and the advantages are many including acquisition speed of 4-5 knots, fixed source - receiver geometry, dense common mid-point (cmp) sampling, real-time quality control, on-board processing and simultaneous acquisition with 2D dual sensor streamer acquisition.

The towed streamer EM system is towed from one vessel. The electric current bi-pole source $800 \mathrm{~m}$ long and is towed at a depth of $10 \mathrm{~m}$. The source runs at 1,500 A, and the source signal is a so-called Optimized Repeated Sequence (ORS), Mattsson et al (2012) with useful frequencies ranging from 0.1 to several Hz. These sequences facilitate a dense set of frequencies, which enhances the inversion performance. Normally, a signal sequence is $120 \mathrm{~s}$ long (one shot) with the source active during the first $100 \mathrm{~s}$ followed by $20 \mathrm{~s}$ of no signal which is used for background noise estimation and noise reduction processing. The streamer with the receiving electrode pairs is $8000 \mathrm{~m}$ long and is towed at a nominal depth of $100 \mathrm{~m}$ if allowed by the water depth. The available offsets are effectively separated by $160 \mathrm{~m}$ and range from 0 to $7600 \mathrm{~m}$. The signal to noise ratio is increased by having long electrode pairs up to $1100 \mathrm{~m}$ for the longest offsets.

The source output current and the electric potential differences at the receivers are measured with the same acquisition electronics. This makes the synchronisation uncertainty negligible and the deconvolution to obtain the frequency responses straightforward. In general the sum of the measurement and navigation uncertainties is below 1\%. Adding the electromagnetic noise to the frequency response uncertainties normally results in total uncertainties below $5 \%$ for the longest offsets and lowest frequencies.

\section{Anisotropic inversion}

The towed streamer EM data allow for anisotropic 2.5D and 3D inversion even though only the inline electric field component is measured. The main reason is the shallow water less than $400-500 \mathrm{~m}$ the system is designed to operate in. The wide frequency and offset ranges also increase the sensitivity to the horizontal resistivity component. This sensitivity decreases with depth below mud line and has shown to be sufficient down to $2200 \mathrm{~m}$ provided that the resistivity bulk structures are big enough. This is demonstrated by a $2.5 \mathrm{D}$ anisotropic inversion on a single line data set over the Alvheim Boa field in the Norh Sea.

The Alvheim Boa field, known as the Alvheim sandstone, is a challenging target due to average size and a depth of burial at 2,100 m below mudline. The reservoir is a turbidite. They are notoriously difficult to evaluate, even in wireline log data, and often show a rather low net-to-gross $(\mathrm{N} / \mathrm{G})$.
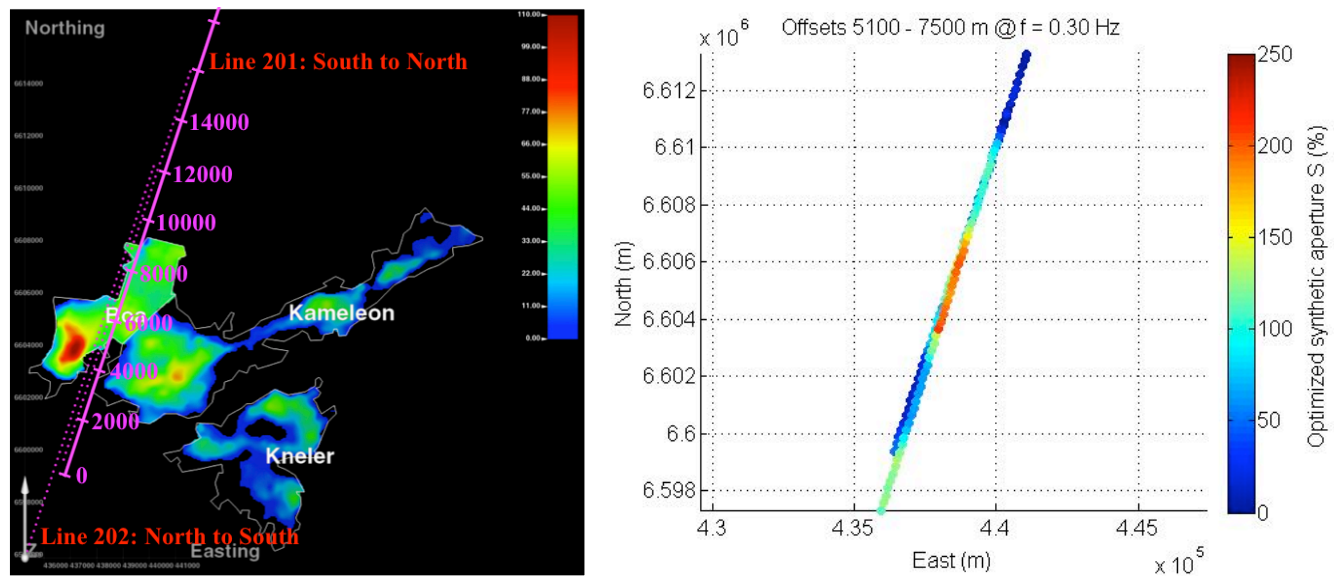

Figure 1 The survey lines close to the depocenter in red (left) and the optimized synthetic aperture frequency response amplitude along the two survey lines 201 and 202 (right). 


\section{Amsterdam '14}

A consistent response in the towed streamer EM synthetic aperture, Mattsson et al (2013), frequency response data is seen in red in the right plot of figure 1 . The response the along the two survey lines of 50 shots each coincide with the depocenter shown as the red area the left plot of figure 1.

The Alvheim - Boa data are inverted with the open-source code MARE2DEM based on a parallel adaptive 2.5D finite element algorithm for the forward modelling and a regularized variant of a Gauss-Newton minimization algorithm for the inversion. Figure 2 shows the inversion result for line 202 where the inversion finite element grid has been guided by the seismic structure in order to conform in shape with the layered horizons. The selected data for all shots in the inversion is: Offsets: $1445,1745,2200,3100,4000,5050,5650,6245,6845,7440 \mathrm{~m}$ and frequencies: $0.15,0.20,0.25$, 0.30 and $0.75 \mathrm{~Hz}$. The misfit after 14 iterations is $2 \%$, which reflects the low uncertainty levels in the data. It is clearly seen that an increase in resistivity is obtained at a depth of $2100 \mathrm{~m}$ and corresponds well with the high resistive depocenter in the sand layer.

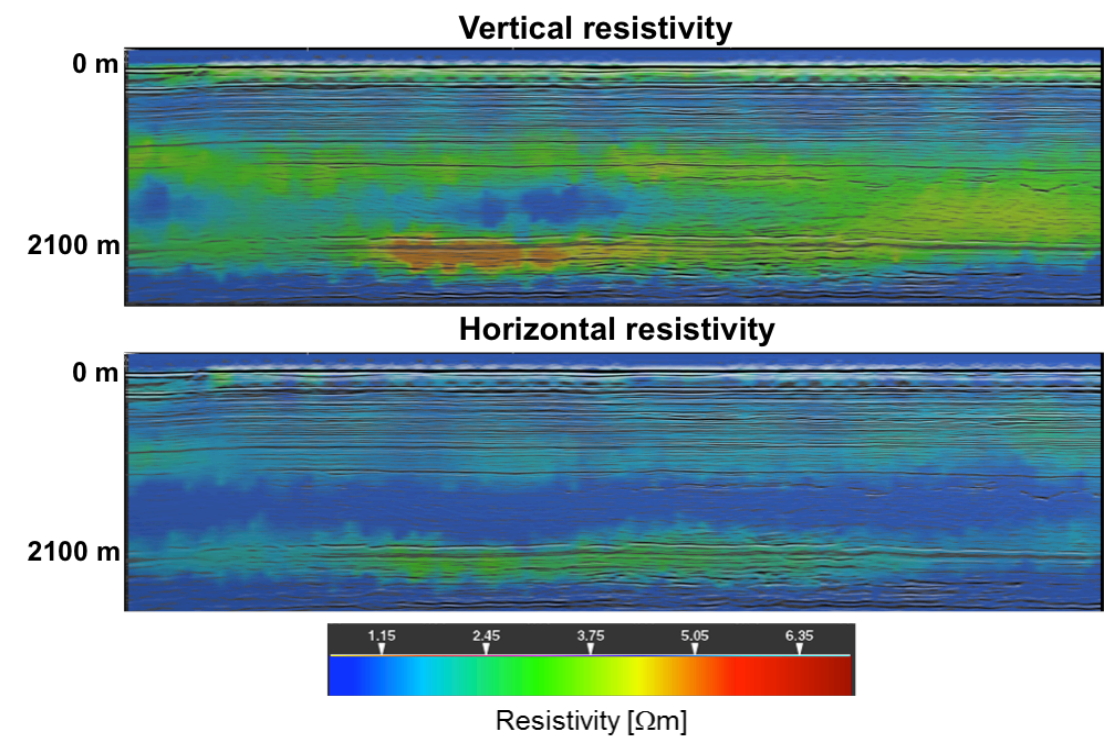

Figure 2 The resulting horizontal (bottom) and vertical (top) resistivity structures after $2.5 D$ inversion. The resistivities structures are plotted on top of seismic structure data.

\section{Conclusions}

The towed streamer EM technology has proven to be a robust and useful CSEM method in shallow waters. It has been proven that the densely sampled frequency response data is suitable for anisotropic inversion of the resistivity structure of the sub surface. The towed streamer EM acquisition technology is particularly efficient in large scale regional surveys with line lengths of several hundred of km even though the it can be used for smaller scale surveys as well. Hence, the towed streamer EM technology is a suitable complement to the conventional node based CSEM technology. In the Alvheim - Boa case, it is demonstrated that 2.5D anisotropic inversion is able to image the high resistive anomaly corresponding with the high resistive depocenter. However, a more dense set of parallel survey lines over the area is necessary in order to obtain a more accurate estimation of the resistivities.

\section{References}

Mattsson, J., Lindqvist, P., Juhasz, R., Björnemo, E. 2012, Noise reduction and error analysis for a towed EM System: 82nd SEG Conference \& Exhibition, Extended Abstracts.

Mattsson, J., Engelmark, F. and Anderson, C. Towed streamer EM: the challenges of sensitivity and anisotropy, First break, volume 31, June 2013. 